\title{
Refractive Uniformity of a Borosilicate Glass After Different Annealing Treatments
}

\author{
Leroy W. Tilton, Fred W. Rosberry, and Florence T. Badger
}

\begin{abstract}
In order to investigate claims that only low holding temperatures are adequate when annealing optical glass for a highly homogeneous product, interferometric tests were made on ten 2-inch cubes of borosilicate glass after an annealing at $515^{\circ} \mathrm{C}$, and then the tests were repeated after the cubes were reannealed, five at $490^{\circ}$ and five at $530^{\circ} \mathrm{C}$. For each of three presentations of the cubes with respect to light paths, contours of differences in refractive index were drawn at intervals of $5 \times 10^{-7}$. It was found that index variations seldom exceeded $\pm 1 \times 10^{-6}$ in this annealed glass. From analyses of the data, it was concluded that there need be little, if any, difference in degree of homogeneity, even if the holding temperature during annealing is 30 or $40^{\circ} \mathrm{C}$ above the lowest feasible value.
\end{abstract}

\section{Introduction}

It is well known that the refractive index and density of glass are functions of annealing temperature $\left[1\right.$, p. 519] ${ }^{1}$. Within limits, the refractive index of a number of silica glasses at room temperature has been found to increase linearly as lower annealing temperatures are selected, provided the holding time is sufficient in each instance to allow the glass to come to a state of approximate structural equilibrium and further that the pieces are sufficiently small to permit cooling to proceed so rapidly that the equilibrium is not appreciably changed thereby. It is also possible to terminate and control these processes of coming to equilibrium by decreasing the holding times at suitable given temperatures and thus obtain lower indices at room temperature than for glass that is annealed at the same temperatures for longer periods.

Because of these possibilities, increased attention is being given to the necessary procedures for adjusting the indices of glass by reannealing at higher or lower temperatures and with shorter or longer holding periods. By such means a higher degree of standardization can be reached in the making of optical glass than has formerly appeared feasible. In this connection, however, the question of relative degrees of homogeneity has properly been raised. If optical glass is arrested, or "fixed", by cooling while in the process of sluggish readjustment from one condition of structural equilibrium to another, is it then as homogeneous as it would be if cooled from almost complete equilibrium at some one annealing temperature? Or is a borosilicate glass, for example, as homogeneous in an equilibrium condition corresponding to an annealing at $530^{\circ}$ or $515^{\circ} \mathrm{C}$ as it would be in its more dense equilibrium condition corresponding to an annealing at $490^{\circ} \mathrm{C}$ ?

According to some views [2] the answers to one or both of the above questions seem to be negative, and there is widespread opinion that optical glass cannot be satisfactorily annealed and homogeneous unless it is as dense and high in refractive index as it is practically possible to achieve by a so-called "full" or "limit" annealing at a rather low holding temperature

To the extent that these views are valid, it seems

${ }^{1}$ Figures in brackets indicate the literature references at the end of this paper. to the writers that their practical application is confined to heat treatments where it may be customary to employ very much higher treating temperatures and much more rapid cooling than is usual in the making of good optical glass. Their full acceptance would lead to longer and unnecessarily expensive programs of fine annealing of optical glass and could preclude much of the contemplated freedom in adjusting the index of refraction for special purposes or for economical standardization.

Such views regarding the necessity of low-temperature annealing may be briefly considered under two main headings. The first of these is degree of homogeneity, and the second is stability. Some of the arguments regarding inhomogeneity seem based on the fact that, during cooling, the surface necessarily cools earlier than the interior. (Such arguments seem to neglect the factor of relative rates of cooling.) Inasmuch as the surface attains higher index while the center is still unchanged, speed in cooling is recommended to insure that the inhomogeneity so produced is kept within permissible limits. Obviously, then, it may be thought that only very low holding temperatures should be used in order that the readjustments of the outer portions shall be so sluggish that they are inconsequential for the purposes for which the glass is intended. This argument, as sometimes presented, seems to overlook the important point that the contemplated inhomogeneity may be in large part only transitory. The interior portions in turn must follow through the same temperature regions, and the center may merely lag with respect to the surface in attaining a higher density. Only difference in cooling time, as between center and surface, during the very early stages of cooling can impress upon homogeneous glass a permanent difference in properties. Since both the center and the surface cool at almost the same rate, after the "steady state" is reached, it follows that (unless the holding temperature is very high) the customary very slow (rather than fast) rates of cooling are initially desirable, at least until the steady state is reached. Slow cooling can be used from any annealing temperature (holding temperature) with the attainment of final homogeneity to almost any desired degree.

On the other hand, in the cooling of glass from a preheating temperature to an adequately high hold- 
ing temperature, it is obvious that time can be saved by initial rapid cooling. Conceivably, also, if the selected holding temperature is unnecessarily high in the annealing range, some time can be saved thereafter by again using a rapid rate of cooling, provided one can know just when to decrease the rate before reaching temperatures at which the lag in attainment of equilibrium demands minimum temperature differences between center and edges until a steady state is reached.

The second point for consideration is the matter of stability at ordinary temperatures. There seems general agreement that the sluggish readjustments that occur in the annealing range proceed more slowly, and more or less exponentially, as the temperature is decreased. Also it is known that many many months of heat treatment are required for the production of very small changes in refractive index at the lower temperatures of the annealing ranges, temperatures that are nevertheless very high indeed compared to room temperature. In the course of 25 years during which experiments of this nature have been in progress at the National Bureau of Standards [1, p. 519] no evidence of instability at room temperature has been found in index of refraction of annealed optical glass of good or even fair quality. Many glass prisms used as standards of refractive index have been measured and remeasured to six decimal places over this 25-year period, and no definite changes have been detected. Anv systematic changes as large as $\pm 5 \times 10^{-6}$ should have been noticed if they occurred. Although most, or all, of these glasses were annealed, some were included that had merely been "pot-cooled." Certainly, none of these prisms were annealed at temperatures so low that maximum attainable indices were even approximated. Therefore, the writers suggest that such changes in refractivity as may occur and be detected in the course of months after extremely severe chilling of glass are, perhaps, not of exactly the same nature as those readjustments that occur in the annealing range and certainly do not need consideration in the fine annealing of optical glass.

Winter's subsequent discussion of annealing problems [3] serves to correct some impressions that were obtainable from her earlier papers. For example, in presenting the "freezing process" it is "emphasized that the cooling rate does not need to undergo any sudden change at $T_{f}$; the variation of temperature can be continuous from $T_{e}$ to room temperature if the cooling rate at each temperature is rapid enough to avoid any further transformation of glass", and the process is called a "method of annealing resulting in glass that is structurally homogeneous although not entirely stabilized and it shows that a considerable gain of time can be realized with respect to the time of limit annealing." Obviously, the lower the holding temperature, the more successfully can this freezing process be applied. But lower holding temperatures are by no means necessary or exclusively desirable. The form of cooling curve from a preheating temperature to a relatively high holding temperature may be selected entirely for economy of time. For this, an initially rapid rate with progressive retardations is reasonable provided the subsequent holding time shall be adequate for structural homogeneity at that particular holding temperature. However, in the practical annealing of optical glass, economy in time of holding will seldom permit selection of a holding temperature so low that sudden cooling can follow without seriously lowering the surface refractivity below that of the central portions. From reasonably high holding temperatures we continue to suggest the usual procedures of cooling slowly at first, so that the rate for surfaces and center may be more nearly equal in the effective annealing region, and then following by very gradual increases in the cooling rate.

\section{Description of Glass Samples and Annealing Procedures}

In order to test the practical importance of differences in annealing, ten 2 -in. cubes of borosilicate glass were prepared from pot glass and polished for inspection as to striae and initial strain, and also for refractive-index measurements with a precision refractometer. They were then all annealed at one time in one furnace by the Glass Section of the National Bureau of Standards, holding at $515^{\circ} \mathrm{C}$ for $12 \mathrm{hr}$ (after a preheating at $555^{\circ} \mathrm{C}$ for $2 \mathrm{hr}$ ) and cooling about as rapidly as advisable after the initial cooling at about $1^{\circ}$ an hour. The cubes (except No. 5) were enclosed in individual boxes of 3/64-in. aluminum and placed in circular array on perforated trays in air only.

After repolishings, tests, and measurements to be described later, cubes $2,4,6,8$, and 10 were reannealed by holding at $530^{\circ} \mathrm{C}$ for $17 \mathrm{hr}$ (after preheating at $555^{\circ} \mathrm{C}$ for $12 \mathrm{hr}$ to insure complete cancellation of all effects of the initial annealing) and cooling from $530^{\circ}$ to $500^{\circ} \mathrm{C}$ at $3 / 4 \mathrm{deg}$ an hour, from $500^{\circ}$ to $475^{\circ} \mathrm{C}$ at $1 \mathrm{deg}$ an hour, etc. In this process these cubes were again enclosed in their individual boxes, which were circularly arranged on small inslulating bricks inside a large aluminum box of 16 -in. diameter and 8 -in. depth, with walls $5 / 8$ in. thick. The large box was in turn separated from the iron box of the furnace by air and by insulating bricks on which it rested.

Cubes $1,3,5,7$, and 9 were similarly arranged in the same furnace, and reannealed by holding at $490^{\circ} \mathrm{C}$ for 15 days (after preheating at $545^{\circ}$ for $10 \mathrm{hr}$ ) and cooling slowly, from $490^{\circ}$ to $425^{\circ}$ at $\frac{1}{2}^{\circ}$ an hour, etc.

The first reannealing, at $530^{\circ} \mathrm{C}$, and its relatively slow cooling should produce glass that is fixed during its incompleted progress from equilibrium conditions at $530^{\circ} \mathrm{C}$ toward conditions at some lower temperatures. This glass is, therefore, about as far below so-called "maximum density" as will ordinarily occur in annealing as practiced for optical glass. The reannealing at $490^{\circ}$ can be expected to produce glass that approximates the room-temperature condition corresponding to an equilibrium 
TABLE 1. Data on 2-in. cubes of borosilicate glase 1

\begin{tabular}{|c|c|c|c|c|c|c|c|c|c|}
\hline \multicolumn{4}{|c|}{ Initial conditions ("pot-cooled") } & \multicolumn{2}{|c|}{ After annealing at } & \multicolumn{4}{|c|}{ After subsequent annealings } \\
\hline \multirow{2}{*}{$\begin{array}{l}\text { Cube } \\
\text { number }\end{array}$} & \multirow{2}{*}{$\begin{array}{l}\text { Refractive } \\
\text { index, } n_{D}\end{array}$} & \multirow{2}{*}{$\begin{array}{c}\text { Strain } \\
\text { birefrin- } \\
\text { gence }\end{array}$} & \multirow{2}{*}{ Striae content } & \multirow{2}{*}{$\begin{array}{c}\Delta n_{D} \\
\left(515^{\circ}\right. \\
\text { minus } \\
\text { pot })\end{array}$} & \multirow{2}{*}{$\begin{array}{c}\text { Strain } \\
\text { birefrin- } \\
\text { gence? }\end{array}$} & \multicolumn{2}{|c|}{$\Delta n_{D}$} & \multicolumn{2}{|c|}{$\begin{array}{l}\text { Strain bire- } \\
\text { frigence 2 }\end{array}$} \\
\hline & & & & & & $490^{\circ}$ minus & $530^{\circ}{ }_{515^{\circ}}$ minus & $490^{\circ}$ & $530^{\circ}$ \\
\hline $\begin{array}{l}1 \ldots \\
2 \ldots \\
3 \\
4 \\
5 \ldots\end{array}$ & $\begin{array}{l}1.51656 \\
1.51655 \\
1.51657 \\
1.51655 \\
1.51657\end{array}$ & $\begin{array}{c}m \mu / c m \\
6 \\
5 \\
8 \\
14 \\
5\end{array}$ & $\begin{array}{l}\text { None } \\
\text { More than cube } 6 . \\
\text { More than cube } 4\end{array}$ & $\begin{array}{c}77 \times 10^{-5} \\
78 \\
76 \\
77 \\
75\end{array}$ & $\begin{array}{c}m \mu / c m \\
3 \\
4 \\
4 \\
3 \\
4 \\
4\end{array}$ & $\frac{74 \times 10^{-5}}{74^{-1}}$ & $\begin{array}{c}-16 \times 10^{-5} \\
-15\end{array}$ & $\begin{array}{c}m \mu / c m \\
2 \\
-3 \\
4 \\
3\end{array}$ & $\begin{array}{c}m \mu / c m \\
-5 \\
4 \\
-\end{array}$ \\
\hline $\begin{array}{l}6 \\
6 \ldots \ldots \\
7 \\
8-\ldots \ldots \\
9 \\
10 \ldots \ldots \\
\end{array}$ & $\begin{array}{l}\text { 1. } 51658 \\
\text { 1. } 51657 \\
\text { 1. } 51655 \\
\text { 1. } 51627 \\
1.51608\end{array}$ & \begin{tabular}{l}
8 \\
9 to 14 \\
\hdashline 8 to 14
\end{tabular} & $\begin{array}{l}\text { Very little } \\
\text { None } \\
\text { Very little }\end{array}$ & $\begin{array}{l}74 \\
74 \\
76 \\
87 \\
81\end{array}$ & $\begin{array}{c}3 \\
- \\
4 \\
4 \\
4\end{array}$ & $\begin{array}{c}76 \\
72 \\
-72\end{array}$ & $\begin{array}{l}-15 \\
-14 \\
-15\end{array}$ & $\begin{array}{c}-3 \\
5 \\
-1\end{array}$ & $\begin{array}{l}4 \\
-5 \\
-5\end{array}$ \\
\hline
\end{tabular}

1 The computed composition of this glass, in percentages by weight, was $\mathrm{SiO}_{2}, 66.6 ; \mathrm{B}_{2} \mathrm{O}_{3}, 12.2 ; \mathrm{Na}_{2} \mathrm{O}, 8.2 ; \mathrm{K}_{2} \mathrm{O}, 12.0 ; \mathrm{ZnO}, 0.5 ; \mathrm{As} \mathrm{O}_{3}, 0.5$

2 These measurements were made by $\mathrm{O}$. H. Grauer in the Bureau's Glass Section by means of a graduated quartz wedge and a set of calibrated strain disks.

at $490^{\circ}$ C. This produces a denser glass with a higher refractivity than is ordinarily obtained in practice. The intital annealing at $515^{\circ} \mathrm{C}$ is of intermediate character, and corresponds more or less to good annealing practice. An important difference between the annealing at $515^{\circ} \mathrm{C}$ and the subsequent annealings was the use of the large aluminum box in each of the reannealings.

The results of preliminary and subsequent examinations of the cubes are given in table 1. Details of the annealing schedules are given in figure 1 . The average indices of these cubes after the pot cooling and after the annealings at $530^{\circ}, 515^{\circ}$, and $490^{\circ} \mathrm{C}$ were $1.51649,1.51708,1.51726$, and 1.51803 , respectively.

\section{Interferometric tests of homogeneity}

In the past it was considered that optical glass was annealed primarily to reduce internal strains and thus prevent birefringence or reduce it within the tolerance limit, say 5 or $10 \mathrm{~m} \mu / \mathrm{cm}$. Now it is realized that it is necessary to anneal primarily to get refractive uniformity throughout the volume of the glass. This can be accomplished by the use of annealing equipment and schedules so designed that the temperature differences within the glass are very small, say small fractions of $1^{\circ} \mathrm{C}$, during the holding period of annealing and the early stages of cooling. Necessarily, then, the glass will be in an unstressed condition as well as homogeneous in refractivity.

\subsection{Qualitative Examinations}

After fine annealings, it is found that glass is almost invariably within the specified birefringence tolerance and the crucial testing, if any, for optical uniformity is done on an interferometer. The simplest and most convenient instrument for such testing of optical glass is the Hilger prism interferometer [4, p. 120] made by application of the Twyman and Green principle to the Michelson interferometer
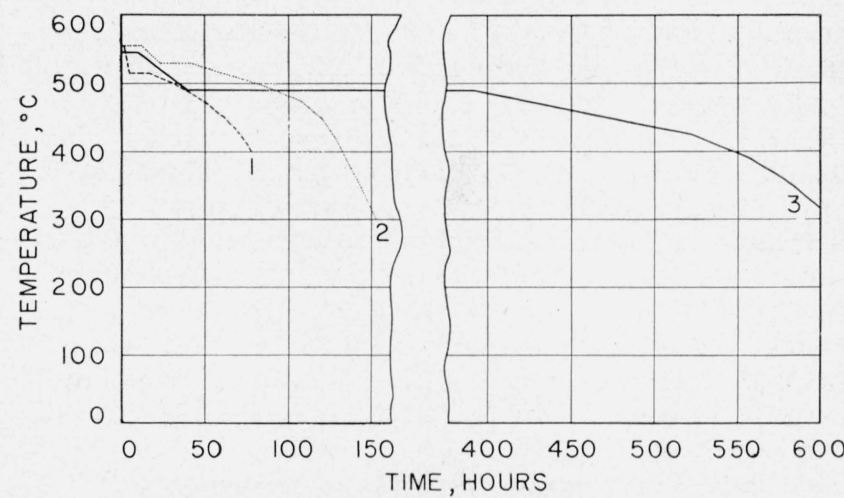

Figure 1. Temperature-time schedules for (1) annealing ten 2 -inch cubes at $515^{\circ} C ;$ (2) reannealing five cubes at $530^{\circ} \mathrm{C}$; and $(3)$ reannealing five cubes at $490^{\circ} \mathrm{C}$.

as arranged for collimated beams. The essential difference from the Michelson is that after reunion at the diagonal, the interfering beams are brought by means of a lens, to a focus for observation or photographic recording.

Unfortunately for the rapid extension of interferometric testing of optical glass, it is necessary to polish two opposite surfaces. If the surfaces are optically plane and the fringes seen through the sample are straight and equispaced, then there is said to be no "error", and it may be concluded that the glass is either (1) homogeneous, (2) its gradients in refractivity are linear, or (3) any nonlinear gradient is parallel to the light beam. If the surfaces are accurately parallel as well as plane, then the component of the optical gradient transverse to the light beams is at once evidenced by the contour of the fringes. The essential planeness of poor surfaces can be effectively achieved by adding a suitable contact liquid and plane parallel plates of almost identical glass. Some samples of borosilicate glass with ground surfaces have been examined interferometrically when combined with methylphthalate and plane parallel (polished) plates as the windows. 
In practice it is assumed that glass showing no error will be satisfactory, even for work of the highest quality. The success of such indefinite and qualitative procedures in the examination of glass means that gradual index gradients in optical glass are usually harmless and approximate the effect of a very weak prism superimposed on the whole optical system as designed and constructed. An important exception is found whenever large prisms are made with internally reflecting surfaces. The Dove erecting prism is a good example. If a linear index gradient exists in a direction normal to the reflecting surface, then the beam transmitted by the prism will be astigmatic, even if the surfaces are perfect.

\subsection{Precise Quantitative Testing}

Occasionally, therefore, when selecting glass for special purposes, such as penta and other large reflecting prisms and the beam splitters and compensators of interferometers, it is desirable to know quantitatively that the existing degree of inhomogeneity is confined within suitable limits. Twyman and Perry [5], in 1922, outlined a method by which this could be computed in the case of nearly plane parallel plates of glass whose thickness would permit interference fringes after reflection at the two polished surfaces. Recent unprecedented demands for very homogeneous glass for large wind-tunnel interferometeis and schlieren benches have stimulated interest in tests of homogeneity, and the new arcs with mercury 198 have made it possible to extend materially the thickness of glass that can be used in interferometry.

If a plate of glass of thickness, $t_{0}$, and index of refraction, $n_{0}$, is placed normally in one arm of an adjusted prism interferometer, the number of fringes, $m_{t}$, seen by transmitted light between points 1 and 2 on the surface of the plate is

$$
m_{t}=\frac{2}{\lambda}\left[t_{0}\left(\Delta n_{1}-\Delta n_{2}\right)+\left(n_{0}-1\right)\left(\Delta t_{1}-\Delta t_{2}\right)\right],
$$

and if the end reflectors of the interferometer are covered, one sees fringes by reflection to the number of

$$
m_{r}=\frac{2}{\lambda}\left[t_{0}\left(\Delta n_{1}-\Delta n_{2}\right)+n_{0}\left(\Delta t_{1}-\Delta t_{2}\right)\right],
$$

where $\Delta t$ and $\Delta n$ are local variations in total thickness and in average refractive index through the plate on lines through the points specified by subscripts.

Twyman [4, p. 136] has indicated a method of plotting contours for the transmission and the reflection fringes and for computing both the inhomogeneity, $\Delta n$, and the difference in glass path, $\Delta t$, according to the equations

$$
\Delta n=\frac{\lambda}{2 t}\left[n m_{t}-(n-1) m_{\tau}\right]
$$

and

$$
\Delta t=\frac{\lambda}{2}\left(m_{r}-m_{t}\right)
$$

between any two points where the two systems of fringes intersect. It is interesting to note that precisely flat surfaces are not required. The writers, in applying this method for accurately investigating the degree of homogeneity in good optical glass, have found it advantageous to photograph both reflection and transmission fringes with a superposed grid of fine wires in order to define numerous points between which comparisons were to be made. The projections of the negatives were measured by means of a comparator and all wire intersections were precisely located with respect to the fringe systems.

Such observations and the requisite computations were carried out with great accuracy for each of the three possible presentations of the cubes on which this paper is written. It was found possible to determine differences in the seventh decimal place of refractive index. In this instance, highly precise data were needed in order to distinguish between, or assess, if possible, the relative merits of different annealing procedures. However, this process is laborious and too slow for acceptance or rejection tests on glass, where in most cases only the fifth decimal in index needs consideration.

\subsection{Rapid Quantitative Testing}

The following procedure, based on observations of the ratio of $m_{r}$ to $m_{t}$ is suggested as feasible, quick, and sufficiently accurate for many cases.

For high-quality optical glass ( $\Delta n$ near zero), and for appreciable values of $\Delta t$, it is evident from eq 1 and 2 that there are more fringes seen by reflection than by transmission, the ratio being $n / n-1$. On the other hand, for poor optical glass with appreciable values of $\Delta n$, and a plate of nearly uniform thickness ( $\Delta_{t}$ being small), the ratio is but little greater than one. For borosilicate glass of near optical quality, the ratio of $m_{r} / m_{t}$ varies from 3.0 to 1.0 , and the mere counting of the number of fringes between two points, neglecting or approximating fractions, is usually sufficient to determine this ratio with adequate precision for useful estimates of $\Delta n$ by an inspection of

\begin{tabular}{|c|c|c|c|c|c|c|c|c|}
\hline \multirow{3}{*}{$t_{0}$} & \multicolumn{8}{|c|}{$m_{r} / m_{t}$} \\
\hline & \multicolumn{2}{|c|}{$\Delta n=1 \times 10^{-7}$} & \multicolumn{2}{|c|}{$\Delta n=1 \times 10^{-6}$} & \multicolumn{2}{|c|}{$\Delta n=1 \times 10^{-5}$} & \multicolumn{2}{|c|}{$\Delta n=1 \times 10^{-4}$} \\
\hline & $\Delta t=10 \lambda$ & $\Delta t=\lambda$ & $\Delta t=10 \lambda$ & $\Delta t=\lambda$ & $\Delta t=10 \lambda$ & $\Delta t=\lambda$ & $\Delta t=10 \lambda$ & $\Delta t=\lambda$ \\
\hline $\mathrm{cm}$ & & & & & & & & \\
\hline 1 & 2. 93 & 2. 93 & 2. 93 & 2. 87 & 2.87 & 2. 43 & 2. 43 & 1. 43 \\
\hline 5 & 2. 93 & 2. 90 & 2. 90 & 2. 64 & 2. 64 & 1. 70 & 1. 70 & 1. 10 \\
\hline 10 & 2. 93 & 2.87 & 2.87 & 2. 43 & 2. 43 & 1. 43 & 1. 43 & 1.05 \\
\hline
\end{tabular}
values of $m_{r} / m_{t}$, such as listed in table 2 .

TABLE 2. Ratios of $m_{r} / m_{t}$ as functions of $t_{0}$ and $\Delta n$ for $\Delta t=$ $1 \lambda$ and $\Delta t=10 \lambda ; M_{0}=1.517$

From eq 1 and 2 , it is evident that $\left(m_{r}-m_{t}\right)=$ $2 \Delta t / \lambda$ and that $m_{t}$, as observed, consists of two parts, $m_{t t}$ owing to $\Delta t$ only, and $m_{t n}$ owing to $\Delta n$ only. As $m_{t t}=\left(n_{0}-1\right)\left(m_{r}-m_{t}\right)$, it is possible to deduce directly from the observations $m_{\mathrm{tn}}=m_{t}-\left(n_{0}-1\right)$ $\left(m_{r}-\dot{m}_{t}\right)$, or, in other works, to obtain the number 
of transmission fringes ascribable solely to inhomogeneity, $\Delta n$ (averaged through a total thickness, $t_{0}$ ), between paths corresponding to points 1 and 2 on the glass surface. For each such fringe solely due to inhomogeneity (that is, under the conditions $m_{t}=1$ and $\Delta t=0$ in eq 1$), \Delta n=\lambda / 2 t_{0}$, and table 3 is computed accordingly.

TABLE 3. Refractive index inhomogeneity, averaged for thickness $t_{0}$, corresponding to a difference of one fringe

\begin{tabular}{|c|c|c|c|c|c|c|c|}
\hline \multirow{2}{*}{$\begin{array}{l}\text { Thickness } \\
\text { of glass, } t_{0}\end{array}$} & \multicolumn{7}{|c|}{$\Delta n$ for various wavelengths } \\
\hline & 7000 & 6500 & 6000 & 5500 & 5000 & 4500 & 4000 \\
\hline $\begin{array}{l}1 \ldots \mathrm{cm} \\
2 \\
2 \\
3 \\
4 \\
5 \\
6\end{array}$ & $\begin{array}{c}35 \times 10^{-6} \\
17 \\
11.7 \\
8.8 \\
7.0 \\
5.8\end{array}$ & $\begin{array}{c}32 \times 10^{-6} \\
16 \\
10.8 \\
8.1 \\
6.5 \\
5.4\end{array}$ & $\begin{array}{c}30 \times 10^{-6} \\
15 \\
10.0 \\
7.5 \\
6.0 \\
5.0\end{array}$ & $\begin{array}{c}27 \times 10^{-6} \\
14 \\
9.2 \\
6.9 \\
5.5 \\
4.6\end{array}$ & $\begin{array}{c}25 \times 10^{-6} \\
12 \\
8.3 \\
6.3 \\
5.0 \\
4.2\end{array}$ & $\begin{array}{c}22 \times 10^{-6} \\
11 \\
7.5 \\
5.6 \\
4.5 \\
3.8\end{array}$ & $\begin{array}{c}20 \times 10^{-6} \\
10 \\
6.7 \\
5.0 \\
4.0 \\
3.3\end{array}$ \\
\hline
\end{tabular}

Similarly, the number of fringes ascribable solely to difference in thickness, $\Delta t$, is $\left(m_{r}-m_{t}\right)$, and for each such fringe difference, $\Delta t=\lambda / 2$.

The glass must be in temperature equilibrium. The surfaces must approximate parallelism so that the fringes are countable, but the degree of planeness, as such, is unimportant. With an isotope mercury source, thicknesses greater than $6 \mathrm{~cm}$ are usable.

Stated as a rule: To determine $\Delta n$ between paths 2 and 1 , count interference fringes, $m_{r}$, formed by reflection and also the transmission fringes, $m_{t}$. Multiply the difference, $\left(m_{r}-m_{t}\right)$ by $(n-1)$, and subtract the product from the observed transmission fringes, $m_{t}$. Multiply the remainder by the appropriate number taken from table 3 .

\section{Details Concerning Precision Measurements}

The source used in examinations of the 2-in. cubes was a krypton tube with a filter suited for transmission of the yellow line of wavelength $5871 \mathrm{~A}$. The cubes were placed on a metal base provided with leveling screws and a vertical frame in which threads were mounted to form a rectangular reference grid of horizontal and vertical lines at intervals of $1 \mathrm{~cm}$. When a nearly perfect cube thus mounted is inserted in one arm of a Hilger interferometer, and adjusted with one of its faces normal to the parallel beam of incident light, one can see by transmission how the glass affects the fringes that would otherwise be present in the interferometer path. One can then compare the transmitted air-plus-glass fringes with the air-only fringes that can at the same time be seea above and at sides of the cube. After shielding the interferometer mirrors, one can view the fringe system that is formed by interference of light that is reflected at the front and rear surfaces of the cube. Figure 2 is a view of the interferometer with a cube in position for photographing the transmission fringes.

For precisely determining the difference in order of interference between the central path and any of the 24 other paths whose points of entrance and emergence are defined by the grid, it was found convenient to make three negatives, which later were projected for readings on a comparator at each of the grid intersections. All exposures were recorded on glass plates with a Panatomic-X emulsion. The plates were developed by the manufacturer's recommended procedure with D-19 developer. The reflection, or glass-only, fringes were first exposed for a duration of 15 minutes. After uncovering the end mirrors, the second plate was exposed for 40 seconds to show

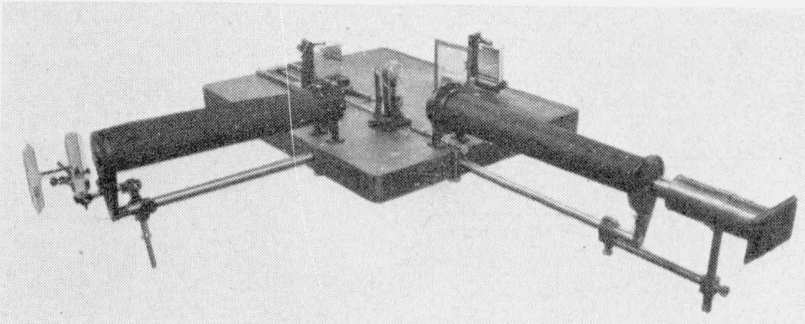

FiguRE 2. Interferometer with glass cube in position for photographing the transmission fringes.

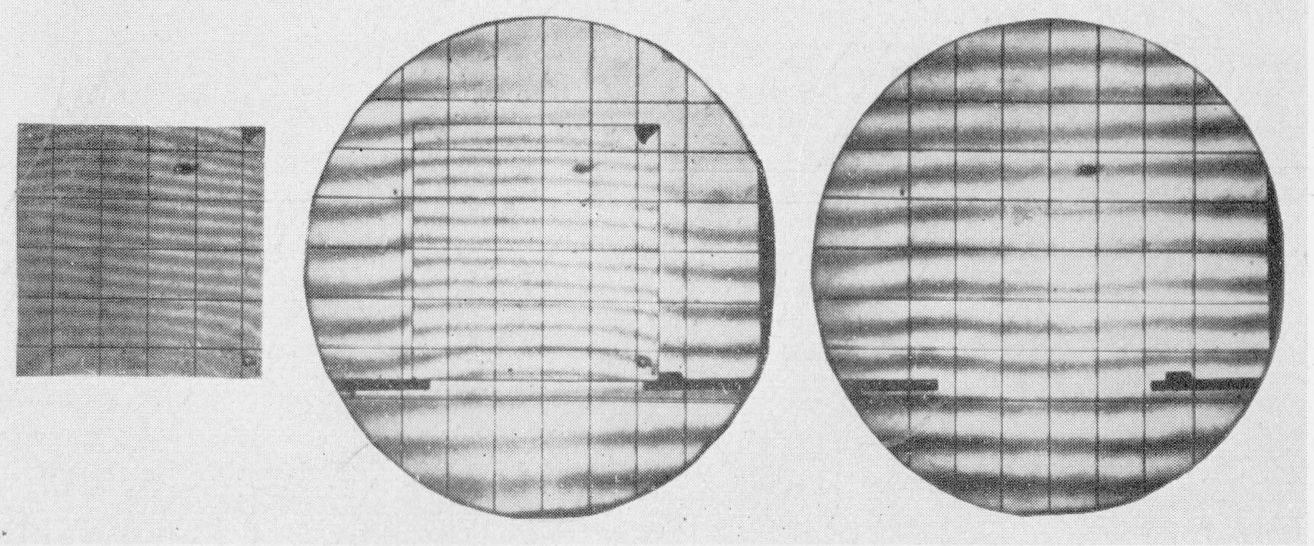

Figure 3. Fringes as photographed for the $B$ presentation of cube 6 after annealing at $515^{\circ} C$.

Left to right: reflection fringes, transmission or air-plus-glass fringes, air-only fringes. 

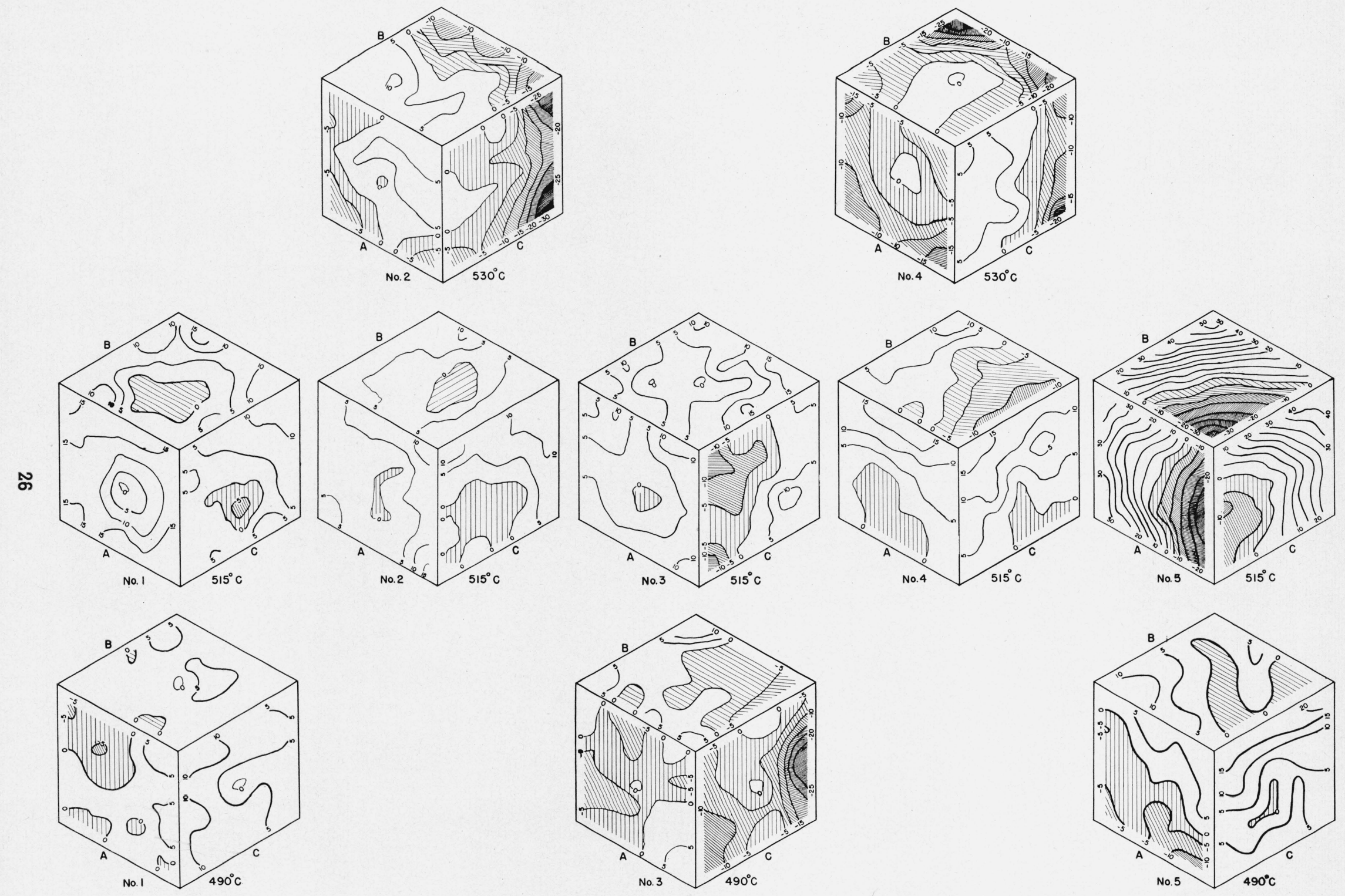

FIGURE 4. Degree of homogeneity after anneaings at different temperatures as expressed by contours with intervals of $5 \times 10^{-7}$ in refractive index. Cubes 1 to 5 . 

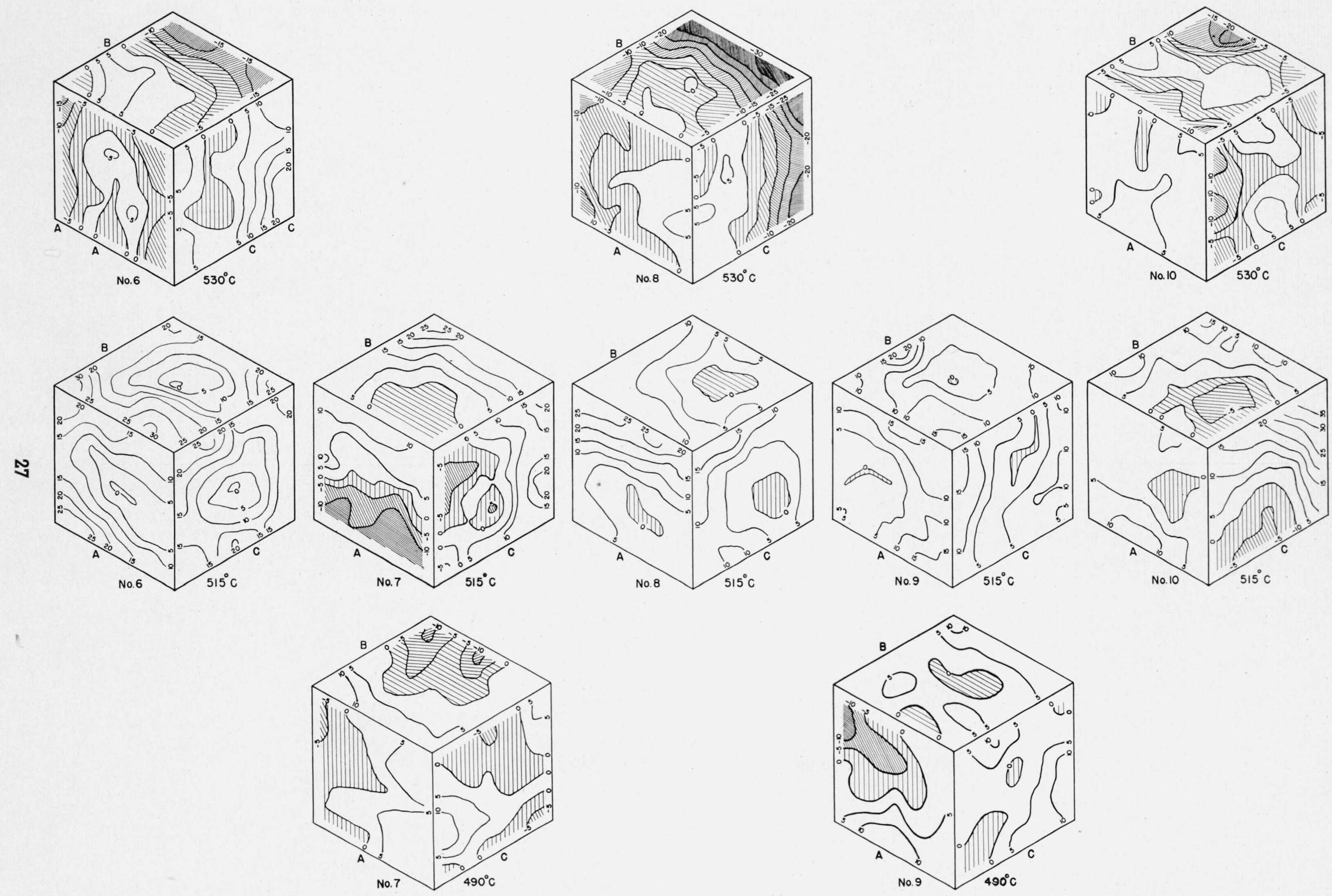

Figure 4. Continued. Cubes 6 to 10. 
the air-plus-glass fringes surrounded by the air-only fringes. If the latter could be precisely set and maintained at one color, it might be possible to operate with only these two exposures, but it is found better to carefully remove the cube after the second exposure and immediately thereafter record the air-only fringes with a 40-second exposure. After reading all three plates at the grid intersections, one compares the air fringes in the second and third exposures for possible shifting of air fringes and makes any necessary corrections. Then one obtains the true transmission fringe readings by subtracting air-only from air-plus-glass readings.

Of course it is necessary to ascertain on each plate the direction in which the whole order of interference increases. Also, the departure from parallelism of opposite faces of the cube must be so adjusted during the polishing that a conveniently measurable number of fringes is seen by reflection. For good glass the geometrical wedge, which can be determined by means of a precision optical gage, determines the direction of increase of the order of interference. In doubtful cases, and for confirmations, one can use local heating at one edge of the cube while the fringe system is being observed.

With a knowledge of the index of refraction and the thickness of the cube, one can use the observed data on order of interference to compute $\Delta n$ by means of eq (3); also, if desired, one computes $\Delta t$ according to eq (4).

\section{Contours of Refractive Inhomogeneity}

For completeness, the interference fringes obtainable in each cube were photographed in each of the three mutually perpendicular presentations. Exposures were made only after the cubes had been in position for at least an hour after handling in a room where the temperature was varying but slowly. The three exposures for a given presentation were made in rapid succession to minimize errors causable by changes in temperature. The fringes obtained for the $B$ presentation of cube 6 after the $515^{\circ} \mathrm{C}$ annealing are shown in figure 3.

Resulting values of $\Delta n$ with respect to the cential path were used in plotting contours at intervals of $5 \times 10^{-7}$ in refractive index. These maps are illustrated in figure 4.

Considering the averages for all three presentations, cubes 1,2 , and 3 were among those that appeared particularly homogeneous after the first annealing at $515^{\circ} \mathrm{C}$, and cubes 6,7 , and 10 were among those least homogeneous. After the reannealing at $490^{\circ} \mathrm{C}$, cube 1 was again very homogeneous, but cube 3 was the least homogeneous of the five in that group. After the reannealing at $530^{\circ} \mathrm{C}$, cube 10 had changed its relative rating and then appeared to be the most homogeneous; cube 2 had also changed, and seemed to be the least homogeneous of that group. Cube 5 was ignored in this connection because, as mentioned in section 2 , its environment, during the first annealing only, was uniquely unfavorable in that no thin aluminum box was provided. Although it had about twice as many contours as the other cubes after the $515^{\circ} \mathrm{C}$ treatment, it became of average condition after the $490^{\circ} \mathrm{C}$ annealing.

The facts given show that we are not dealing primarily with fixed chemical inhomogeneity in these cubes but with temperature effects impressible on the medium in a varying manner. As this borosilicate glass has a refractive sensitivity of about $5 \times 10^{-5} / 1 \mathrm{deg} \mathrm{C}$ in annealing temperature, it is indicated that the gradients in the annealing furnace were about $0.04 \mathrm{deg} \mathrm{C} / \mathrm{in}$. lower inside the thin aluminum boxes than outside. Also, from the moreor-less-pronounced changes in comparative ratings of the cubes, it may be concluded that the gradients inside the boxes may have varied from box to box during a given annealing.

In most of the contour maps the evidenced inhomogeneity is so small and evenly distributed that there is little readily detectible systematic arrangement. In cube 5 after the $515^{\circ}$ treatment, however, all three component maps agree in indicating a higher effective annealing temperature near the edge shown in the foreground. Similarly, cubes 4 and 8 after the $530^{\circ} \mathrm{C}$ treatment evidence higher effective annealing temperatures at their rear edges. But to a considerable extent these contours lack marked systematic arrangement, and this suggests that any inhomogeneity caused by furnace gradients during holding times or by differences in time of cooling of surface and interior are probably masked. Unquestionably, there may exist in these contour maps some masking effects of room-temperature gradients at the time the interference fringes were photographed. It was on this account that after initial equilibrium, all three exposures were taken in close sequence for each presentation and all three presentations for each cube were completed in as short a time as possible. However, since the change in refractive index of this borosilicate glass is only $17 \times 10^{-7} / 1$ deg $\mathrm{C}$ change in room temperature, it is evident that room-temperature variations would have to cause gradients of from $0.5 \mathrm{deg}$ to $1.5 \mathrm{deg}$ $\mathrm{C} /$ in. within the glass cubes in order wholly to account for the apparent inhomogeneities.

There are other reasons why it cannot be assumed that these contours are largely accidental. In all cases the photographic negatives of interference fringes were measured by two observers and the requisite computations made independently. In general, the disagreements are small in the seventh decimal place of $\Delta n$, and only a veraged results were used for plotting the contours. In many of these maps the run of adjacent contours gives internal evidence of precision well within the limits of one contour interval. Considering all data and the facts mentioned in this analysis, and although room temperature as well as furnace gradients may have somewhat influenced the final contours, it is certain that all of these cubes are very homogeneous. As will be seen in the following section, these data can be averaged to minimize the aspects of accidental character, and then cnalyzed to show clearly some 


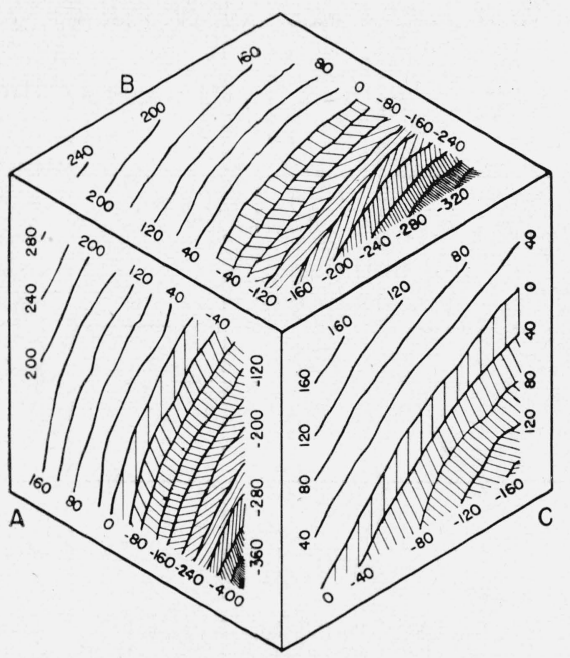

G No.I

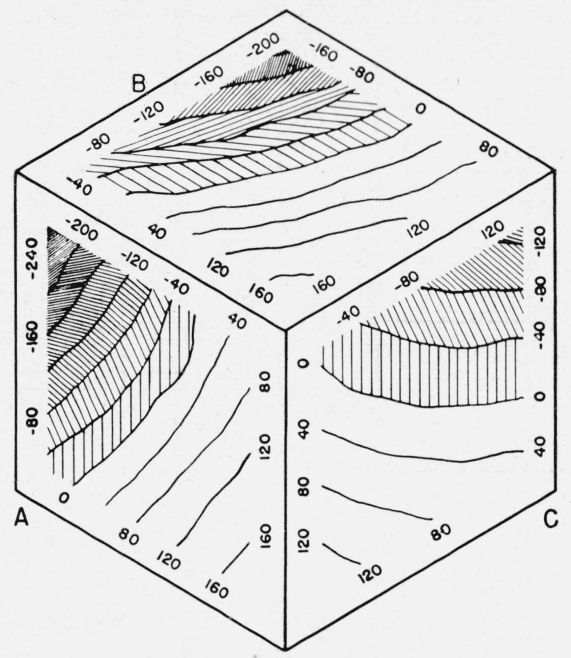

G No. 2

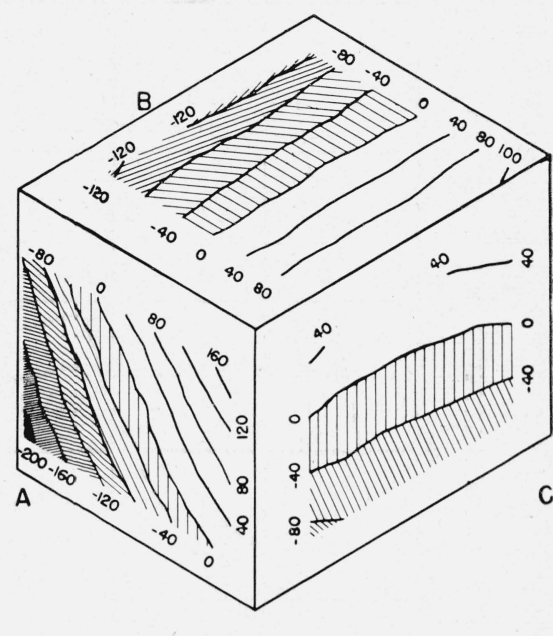

REJECT

Figure 5. Inhomogeneity of two cubes from cargo of German submarine and one sample of domestic optical glass rejected Contour interval $40 \times 10^{-7}$ in refractive index. as unsatisfactory.

systematic effects that are reasonably ascribable to temperature conditions during the annealings.

In contrast with the homogeneity represented in figure 4 , there are presented in figure 5 some similar results on cubes fashioned from German optical glass (annealing grade unknown) taken from the cargo of a submarine that was intercepted during World War II on its way to Japan, and also the results on a sample of domestic optical glass that was rejected because it had been unsa tisfactorily annealed. Note that in the figure 5 diagrams the contour interval is $40 \times 10^{-7}$, or eight times as large as in the cubes of figure 4 .

\section{Comparative Results for Different Annealings}

For comparative purposes, a method of obtaining an a verage estimate of homogeneity for all cubes used in each annealing is desirable, and it is important and convenient to consider asvmmetry as well as the radially distributed symmetrical changes in refractivity. As a preliminary for both considerations, the 24 observed values of $n$ were considered, for each of the three presentations of each cube, according to their sign and their distance from the central path. As will be evident from figure 6, the paths are elements of the surfaces of five cylinders whose projections are shown as circles on a cube face. The radii are $1, \sqrt{2}, 2, \sqrt{5}$, and $2 \sqrt{2} \mathrm{~cm}$, with four values of $\Delta n$ corresponding to each cylinder except the next largest, which has eight values. In each circle the maximum difference in values of $\Delta n$ between diametrically opposite elements was taken as an arbitrary measure of the asymmetrical inhomogeneity for the corresponding eylindrical zone of that particular presentation of the cube. Such values averaged for

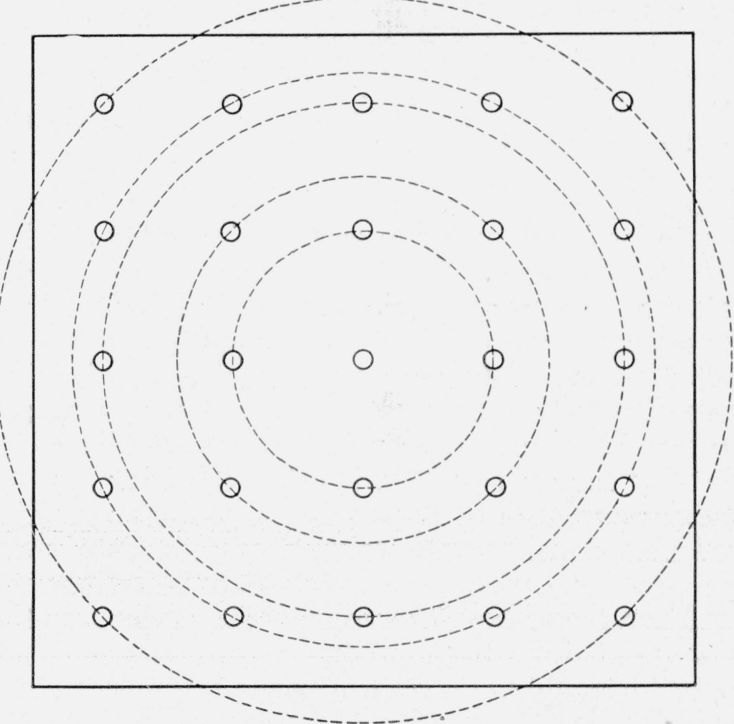

FIgURE 6. Schematic cylindrical shells for use in analysis of data on degree of homogeneity.

The refractivities along 24 elements of five concentric cylinders were determined with respect to the axial path at center. In each shell the maximum difference in index between diametrically opposite elements is an arbitrary measure of the in index between diametrically opposite elements is an arbitra
asymmetrical inhomogeneity at five distances from the center.

three presentations can be plotted against radii for a measure of the maximum asymmetrical inhomogeneity of each cube after each annealing. Such a curve, a veraged for the initial data on all numbered cubes except 5, is characteristic of the first annealing. Similarly, later data on cubes $1,3,7$, and 9 yield a curve for the reannealing at $490^{\circ}$, and cubes $2,4,6$, 8 , and 10 provide for the reannealing at $530^{\circ} \mathrm{C}$. These curves of zonal variation in refractivity, figure 7 , show the asymmetrical distribution of the existing inhomogeneities. 


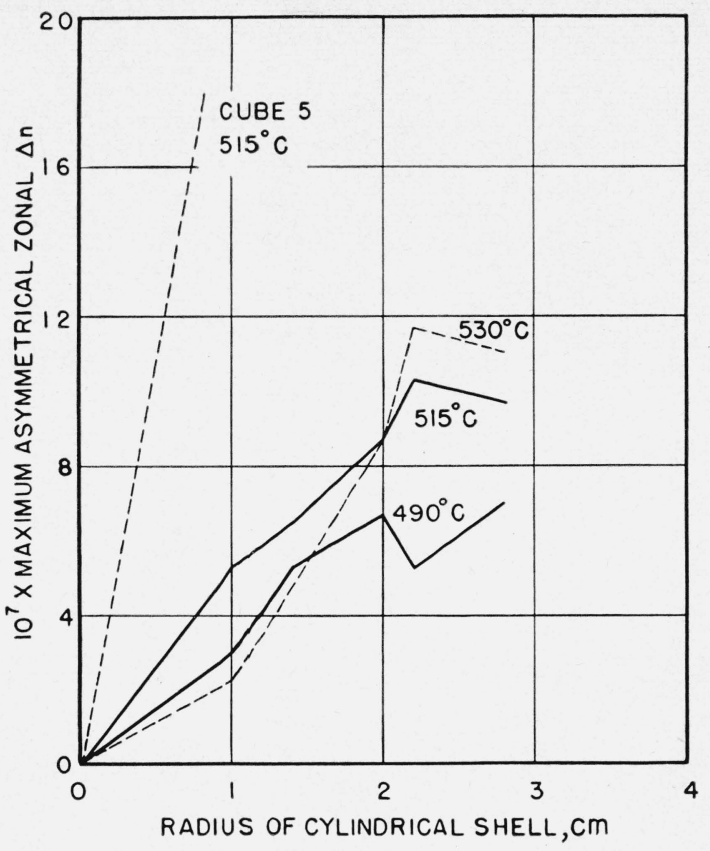

Figure 7. Effect of temperature gradients during holding periods of annealing.

Since the orientation of asymmetry does not persist in given cubes from one annealing to another (fig. 4), the cause is not chemical in nature but merely residual thermal gradients of the order of $0.01 \mathrm{deg} \mathrm{C}$ per inch during annealings Concerning cube 5 , see p. 26.

If for two different annealings the asymmetry does not persist in almost the same orientation within a given cube, then it may be concluded that the effective causes are not chemical in origin but principally thermal in nature. This is certainly the case for cubes 1 to 10 . Linear gradients of refractive index across the cubes cause straightline curves of zonal heterogeneity, and larger index gradients are indicated by larger angles with the $\mathrm{X}$-axis. An outstanding example of approximately linear gradients through a considerable volume of glass is given by cube 5 after the annealing at $515^{\circ} \mathrm{C}$ without its aluminum cover box, the values being fully five times the average for other cubes. From figure 7 , then, it may be concluded that furnace temperature gradients, inside the aluminum covers, were reduced to about $0.01 \mathrm{deg} \mathrm{C} / \mathrm{in}$. during this annealing, and that without the thin aluminum covers the gradients might have been $0.05 \mathrm{deg} \mathrm{C} / \mathrm{in}$.

Insofar as asymmetrical inhomogeneity is concerned, a valid comparison of the different annealings could be made by measuring the areas under the curves of figure 7. A satisfactory approximation is given by comparing averages of the ordinates for the observed radii. Excluding cube 5 for the initial annealing, the averaged ordinates are 6,8 , and $8 \times 10^{-7}$ for the annealings at $490^{\circ}, 515^{\circ}$, and $530^{\circ} \mathrm{C}$ respectively. These differences in homogeneity between annealings are, therefore, so small that their significance is questionable. It would be necessary to conduct further experiments if the validity of differences of \pm 1 or $2 \times 10^{-7}$ in index over 2 in. of glass patch is to be established. Thus, insofar as

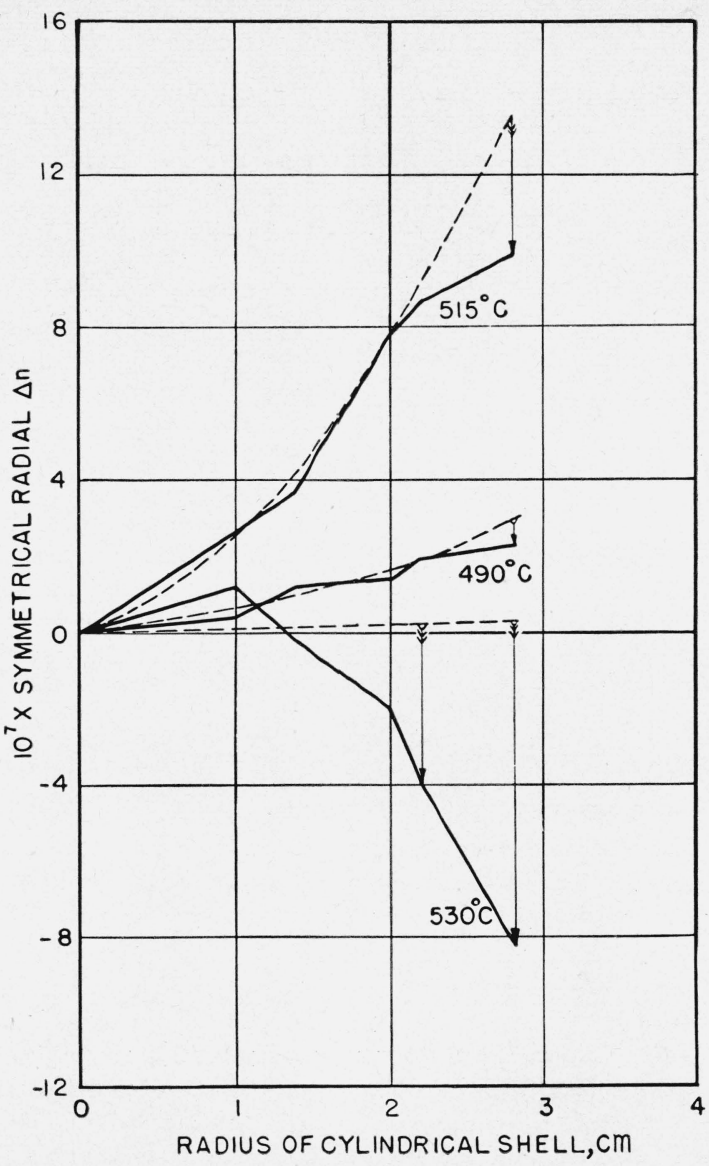

Figure. 8. Effects attributable to duration of holding time and to rate of cooling.

Inadequate holding at medium and low annealing temperatures can (if preceded by preheating) cause relatively lower index (less increase) at centers. The arrow indicate estimates of the relatively greater reduction of index (less increase) at edges during cooling.

asymmetry of inhomogeneity is concerned, the data of figure 7 show that the reannealing at the comparatively high temperature of $530^{\circ}$ is essentially the equivalent of the one at the comparatively low temperature of $490^{\circ} \mathrm{C}$. This may mean, chiefly, that the furnace-temperature gradients can be, and were, essentially the same for each of these annealings.

The curves of figure 7 give no indication of the radial gradients in index that may exist, symmetrically, from center to faces of the cubes. In order to compare the annealings in this respect, figure 8 was prepared. Here, as in figure 7 , the ordinates are values of $\Delta n$ averaged for the same cylindrical shells but for this symmetrical result, a simple algebraic average of $\Delta n$ is used to represent the refractivity of each shell as compared with its axis. For the annealing at $530^{\circ} \mathrm{C}$, it is evident that the outer portions of the glass cooled faster than the center with consequent lower index corresponding to a slightly higher $\left(0.02^{\circ} \mathrm{C}\right)$ equilibrium temperature condition. For the annealing at $515^{\circ} \mathrm{C}$ the $12-\mathrm{hr}$ holding period seems to have been inadequate for raising the index in the center as high as at the edge. A faster cooling 


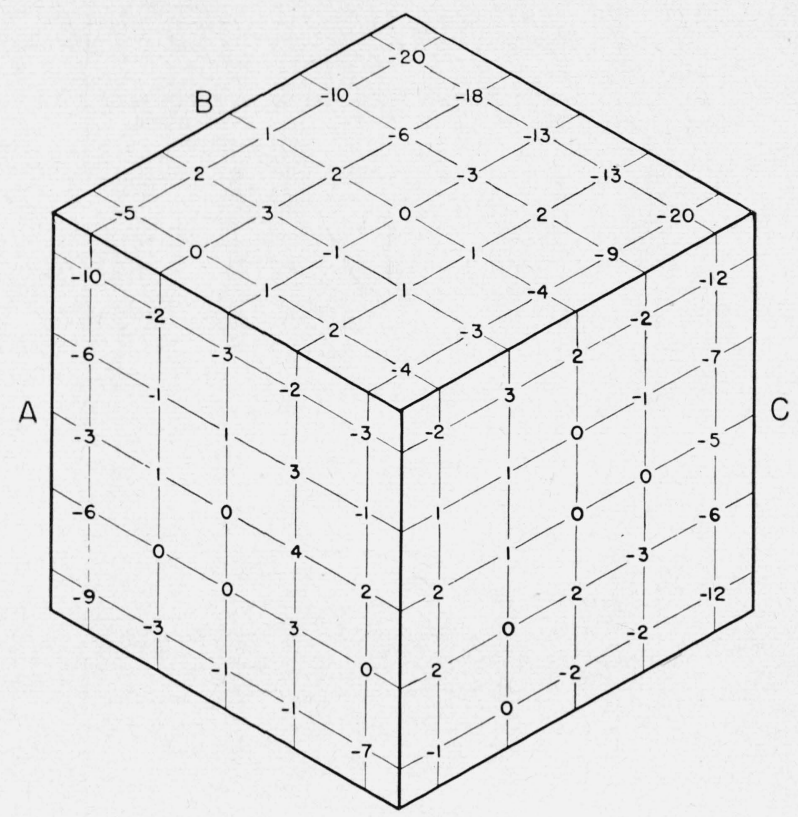

(a)

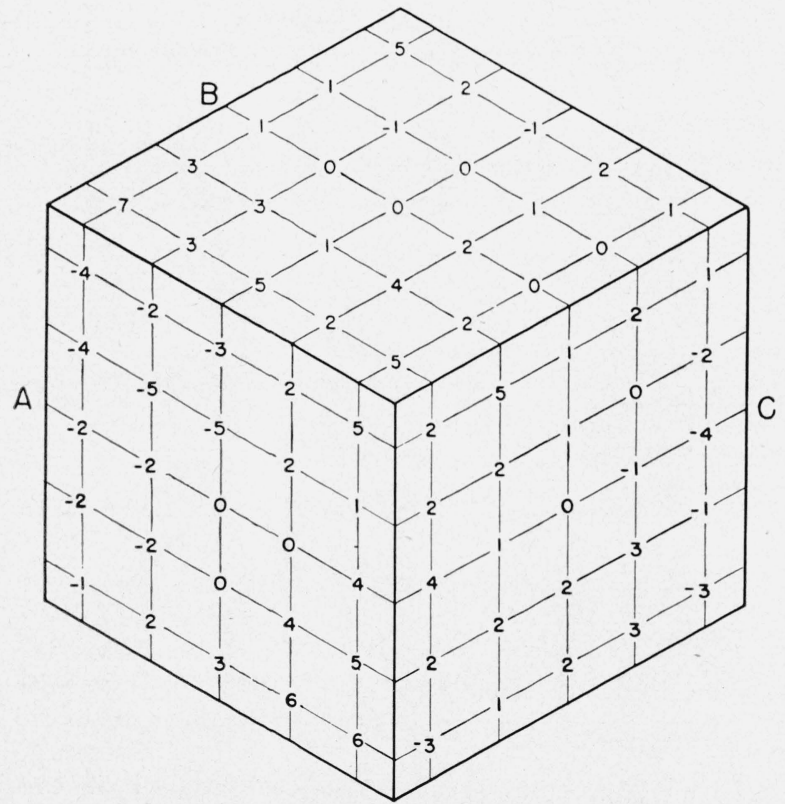

(C)

rate would have, to some extent, offset this index difference. At $490^{\circ} \mathrm{C}$ the holding period of 15 days was probably inadequate, but the cooling rate was almost right in order to compensate therefor.

The actual average distributions of the inhomogeneities after the three annealings are shown in the composite cubes of figure 9 . The important fact is that all three of these annealings produce glass uniform in index within approximately $\pm 10 \times 10^{-7}$. Such glass can be considered practically perfect, insofar as the users of optical glass are concerned, for any elements that can be manufactured from 2-in.

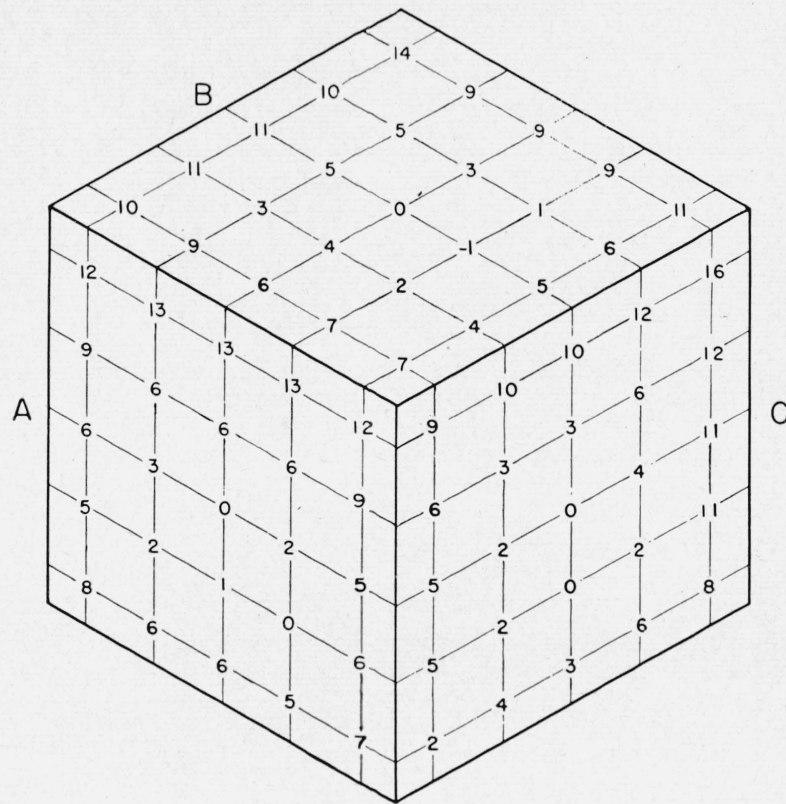

(b)
Figure 9

(a) Homogeneity expressed as $\Delta n \times 10^{-7}$ for composite of five cubes $(2,4,6,8$, and 10$)$ annealed at $530^{\circ} \mathrm{C}$. The initial cooling rate of $3 / 4 \mathrm{deg}$ per hour was slightly too rapid so that the edges cooled faster than the center, with consequent higher effective annealing temperature and lower refractive index.

(b) Homogeneity expressed as $\Delta n \times 10^{-7}$ for composite of nine cubes (1 to 10 , except 5 ) annealed at $515^{\circ} \mathrm{C}$. Following preheating, the holding period of 12 hours was probably not entirely adequate to raise the index at center as high as the edges. The cooling rate of $1^{\circ} \mathrm{C}$ per hour was not sufficiently rapid to entirely compensate by lowering the index at the edges.

(c) Homogeneity expressed as $\Delta n \times 10^{-7}$ for composite of four cubes $(1,3,7,9)$ annealed at $490^{\circ} \mathrm{C}$. The holding time of 15 days was almost adequate for raising the central index as high as that at the edge, and the initial cooling rate of $1 / 2 \operatorname{deg} \mathrm{C}$ per hour was so slow that the index at the edges was not materially lowered cubes. Even for wavelengths as short as $0.4 \mu$ the distortions that could be imposed on wave fronts cannot exceed Rayleigh's limit of $\lambda / 4$, unless the paths in glass of this quality are longer than $5 \mathrm{~cm}$.

The conclusion that borosilicate glass homogeneous within $\pm 1 \times 10^{-6}$ in refractive index can be obtained by annealings in which the holding temperature is 30 or 40 deg $\mathrm{C}$ above the lowest feasible annealing temperature is contrary to certain ideas that have been widely presented and have obtained some credence in recent years regarding an alleged practical superiority resulting from annealing at very low 
temperatures, and the alleged necessity of obtaining maximum "compaction" in order to obtain desirable homogeneity. On the other hand, the results obtained in this investigation are in full accord with ideas expressed by Tool [6] and associates concerning the possibilities of making useful adjustments in the refractive indices of very homogeneous optical glass by the choice, within limits, of suitable annealing temperatures.

\section{References}

[1] Arthur Q Tool, Leroy W. Tilton, and James B. Saunders, J. Research NBS 38, 519 (1947) R P1793.

[2] A. Winter, J. Am. Ceram. Soc. 26, 189-200, 277-284 (1943).
[3] A. Winter, J. Am. Ceram. Soc. 27, 266-274 (1944).

[4] F. Twyman, Prism and lens making (Adam Hilger, Ltd., London).

[5] F. Twyman and J. W. Perry, Proc. Phys. Soc. London, 34, 151 (1922)

[6] A. Q. Tool and L. W. Tilton, Annealing as a means of producing desired refractivity changes in glass, read at meeting of Am. Ceram. Soc., Washington D. C. (1932). A. Q. Tool, L. W. Tilton, and J. B. Saunders, Effect of heat treatment on the refractive index of glass, read at meeting of Opt. Soc. Am., Corning, N. Y. (1937). Changes caused in the Refractivity and density of glass by annealing, J. Research NBS 38, 519 (1947) RP1793.

Washington, January 31, 1952. 